\title{
Preparation and Characterization of Asymmetric Ultrafiltration Membrane for Lysozyme Separation: Effect of Polymer Concentration
}

\author{
A. Nora'aini ${ }^{1}$, H. Sofiah ${ }^{2}$, A. Asmadi ${ }^{3} \&$ M. Abdul Wahab ${ }^{4}$ \\ $1,2.83$ Department of Engineering Science, Faculty of Science and Technology, University Malaysia Terengganu, \\ 21030 Kutala Terengganu, Malaysia \\ ${ }^{4}$ Department of Chemical Engineering \& Process, Faculty of Engineering, Universiti Kebangsaan Malaysia, \\ 43000, Bangi, Selangor
}

\begin{abstract}
Penetrations of membrane technology in the downstream processing of protein production are now well accepted due its effectiveness and ability to generate high yield of product. This study aimed to investigate the effects of polymer concentrations on morphology and performance of ultrafiltration membranes for lysozyne separation. Three membrane solutions with different polymer concentration of Polyehtersulfone/N-Methyl pyrolidone/water with $15 \% \mathrm{w} / \mathrm{w}, 17 \% \mathrm{w} / \mathrm{W}$ and $19 \% \mathrm{w} / \mathrm{w}(15 \%$ [UF 15], 17\% [UF 17] and 19\% [UF 19]) were used to prepare asymmetric flat sheets membranes via a simple dry/wet phase inversion techniquc. The data obtained shows that the permeability coefficient decreased from $10.9 \times 10^{-6}$ to $0.44 \times 10^{-6} \mathrm{~m}^{3} / \mathrm{m}^{2}$.s. bar with increasing polymer concentrations. UF 15 membrane discovers the highest flux and lysozyme transmission, consequently piomoted this membrane as an optimum membrane for lysozyme separation. This study proves that the polymer concentration was greatly affected the membrane performance and struct urai properties consecutively, promoted an improvement of UF membrane capability for lysozyme separation.
\end{abstract}

Keywords: Membrane, ultrafiltration, lysozyme, lysozyme purification

\subsection{INTRODUCTION}

Ultrafiltration has a wide variety of application, ranging from the processing of biological macromolecules, electrocoat paint recovery, enzyme and pharmaceutical preparations to wastewater treatment [1]. Some of the major applications are the fractionation of nucleic acids, concentration of macromolecules, dialfiltration, removal of cells and debris from fermentations broth, virus removal from therapeutic products, harvesting of biomass and effluent treatment [2] and also in many process engineering with significant technical and commercial impact.

* Corresponding to: A. Nora'aini (emai1: noraaini@umt.edu. my)
Ultrafiltration membranes, based on variety of synthetic polymers, have high thermal stability, chemical resistivity, and restricted the use of fairly harsh cleaning chemicals [3] [4]. This type of membrane demonstrates a heterogeneous morphology which consists of a thin skin with a thickness between $0.1-0.5 \mu \mathrm{m}$ and a porous supporting sub layer with a thickness between $50-150 \mu \mathrm{m}[5]$. They show a greater permeation rate than symmetric membranes of comparable thickness of the actual barrier layer [6]. The asymmetric structure of UF membrane gives the membranes its required mechanical strength (which is provided by the support layer) along with its desired separation properties (which are governed by the skin layer). It is recognised that the separation properties of porous membranes 
also depends on their physical properties including porosity and pore size distributions [7].

Formulation of membrane material and material content greatly influenced the asymmetric UF membrane at the first stage of membrane making. Inorganic or organic polymer, solvent, non solvent and additive composition will give significant effect on fabricated membranes. The membrane characteristics affected by the membrane composition are morphology, pore size, thickness, viscosity of membrane solution and also gelation time which overall related to the membrane performance in term of selectivity and productivity [8]. Changes in the solution structure by polymer composition affects the performance of the resultant membrane as it plays a role in improving macrovoids structure and thickness which lead to influence the membrane permeability and productivity. Both separation performance and the permeate flux of ultrafiltration membrane are conceptually related to its pores pore size. High transmission obtained whenever the size of the solute is small relative to the pore size of the membrane.

Controlling the pore size and creating suitable pore size distributions of membranes is important in specialized industrial applications. When water - soluble polymers are used in the solution film. during phase inversion process, the hydrophilic polymers starts to be dissolved by water and the sites polymers existed become micropores. The solvent in the casting solution film is exchanged with water and phase separation occurs in the film. It is usually turns out to be a characteristic morphology of asymmetric membrane showing a dense top layer and porous sub-layer. The sub-layer which consists of macrovoids, pores and micropores is governed by variation in the casting dope solutions such has concentration, temperature and organic and inorganic additives [9].

PES is widely used as UF membrane material due to its high rigidity, creep resistance, good thermal and dimensional stabilities [10]. A lower polymer concentration and strong interaction between water and PES results in the growth of thin polymer layer [11]. Water is claimed to be strong non-solvent for PES polymer and coagulation process will occur faster when the polymer solution is brought into contact with water and the finger-like structure formed. Large fingerlike macrovoids is generally formed when the coagulation process is faster, whereas the slow coagulation rate results in a porous sponge-like structure [12].

To date, membrane properties have been tailored and adjusted to the specific task in order to enjoy numerous industrial applications with their advantages, including separation and purification of protein molecule. Lysozyme is one of the most significant proteins which gained a great interest in biotechnology research and industries. It is known as the smallest protein in egg albumin with the concentration that make up approximately $3.4 \%$ of total eggs white protein [13]. Lysozyme was being a significant enzyme for different applications including as a food additive in milk products, a cell-disrupting agent for extraction of bacterial intracellular products, as an antibacterial agent in ophthalmologic preparations and in wine production processes and also been used in the treatment of HIV infection. Besides, lysozyme is also used to be a drug for treatment of ulcers and infections [14] since it can performs antibiotic function [15] for the human body. The low content of lysozyme in chicken egg white makes its purification process becomes challenge and complicated either in batch or large scale system. A large quantity of raw material has to be processed in order to get a reasonable amount of pure lysozyme. Therefore, an efficient, large scale protein purification process is required to apply for lysozyme purification [16].

In the present study, the effects of polymer concentration on flat sheet asymmetric ultrafiltration membranes were examined. The suitability of three membranes formulations from 15 to $19 \mathrm{wt}$ \% for the separation of lysozyme were assessed using lysozyme single solution. The objective was further validated the characteristic of the best chosen formulation membrane by attempting the membrane morphology and its molecular weight cut off.

\subsection{METHODOLOGY}

\subsection{Materials}

All materials used were of analytical grades. The membranes were fabricated from a ternary casting solution which consisted of polyethersulfone 
(supplied by Merck) as polymer, N-methyl-2pyrrolidone (NMP) (supplied by Merck) as a solvent and water $\left(\mathrm{H}_{2} \mathrm{O}\right)$ as a non-solvent. Lysozyme ( $M w=14700$ Dalton) purchased from Sigma Aldrich has been used for the evaluation of membrane performance.

\subsection{Membrane Preparation}

The membranes were prepared using three different ternary dope formulations of casting solutions as shown in Table 1. Asymmetric UF membranes were fabricated via phase-inversion techniques using semi-automated electrical casting machine at an approximately constant shear rate of $200 \mathrm{~s}^{-1}$. Distilled water was used as the first coagulation bath to induce the polymer precipitation for about 24 hours. Subsequently, the membrane was immersed in methanol (supplied by Merclk) for about 8 hours to ensure the excess solvents were totally removed and to strengthen the molecules structure build in the membrane. The membrane was dried at room temperature for 24 hours before being used.

Table 1 Ternary dope formulation

\begin{tabular}{ccc}
\hline PES (wt.\%) & NVP (wt.\%) & Water (wt.\%) \\
\hline 15 & 77 & 8 \\
17 & 77 & 6 \\
19 & 74 & 7 \\
\hline
\end{tabular}

\subsection{Membrane Characterization}

\subsubsection{Permeation with Pure Water and Lysozyme}

All permeation experiments were carried out using dead end cells with $300 \mathrm{ml}$ processing volume and effective permeation membrane area of 14.6 . Distilled water was used for pure water permeation to obtain pure water permeability and to ensure the membrane stability. For lysozyme permeation, $500 \mathrm{mg} / \mathrm{L}$ lysozyme solution was prepared in sodium chloride $(0.1 \mathrm{M})$ at room temperature. Feed pressure was controlled in the range of 2 to 10 bars by using compressed nitrogen and $10 \mathrm{ml}$ of permeate was collected. The absorbance of feed, permeate and retentate of lysozyme permeation were analysed by UV-Vis spectrophotometer (Hitachi U-2000) at wavelength $280 \mathrm{~nm}$. The average data of three replicates were reported.

\subsubsection{Determination of Membrane Morphology}

The Scanning Microscopy Electron (SEM) (JSM $\mathrm{P} / \mathrm{N}$ HP475 model) has been used to inspect the cross section of the fabricated membranes. For this purpose, the membrane samples were fractured in liquid nitrogen and sputtered with gold, before transterring them under the microscope.

\subsubsection{Determination of Molecular Weight Cut-Off}

A series of protein (myoglobin [17kD], ovalbumin [40kD], Pepsin [35kD] and BSA [66 kD]) with different molecular weights were used for rejection study to determine the molecular weighit-cut off of the fabricated membranes. Feed and permeate concentrations were analysed using UV-vis spectrophotometer at wavelength $280 \mathrm{~nm}$. Percentage of rejection was calculated using equation 2.1 .

$$
\% \text { Solute Rejection }=\left[1-\left(C_{p} / C_{p}\right)\right] \times 100
$$

where $C_{p}$ and $C_{f}$ are the concentration of permeate and feed, respectively.

\subsection{RESULT AND DISCUSSION}

\subsection{Permeability Coefficient}

Pure water permeation has been implemented for all fabricated membranes at five different pressures from 2 to 10 bars. The graph of filtrate flux versus pressure for different polymer concentrations of UF membrane is displayed in Figure 1.

All membranes show linear function for increased of applied pressure from 2 to 10 bars and this profile was followed the Hagen-Poiseuille equation which stated that the water flux incensement is proportional to the increase of applied pressure.

$$
I_{v}=P_{m} \Delta P
$$




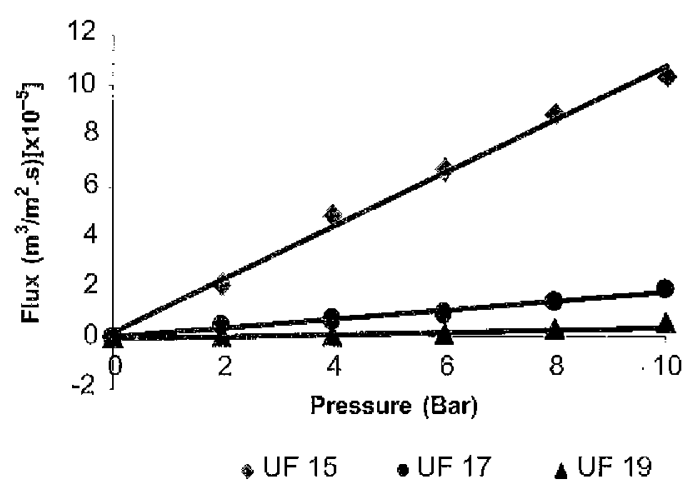

Figure 1 Pure water flux of three fabricated membranes

The values of permeability coefficient for the three membranes have shown in Table 2. The UF 15 membranc shows the greatest permeability coefficient within the range of porous membrane which was $10.9 \times 10^{-6} \mathrm{~m}^{3} / \mathrm{m}^{2}$.s. Permeability coefficient decreased with increased polymer concentration in the membrane solution. This was clearly seen since the permeability coeflicient for UF 17 and UF 19 were reduced to $1.82 \times 10^{-6}$ and $0.44 \times 10^{-6} \mathrm{~m}^{3} / \mathrm{m}^{2}$.s respectively.

The results induce that UF 15 membrane was a thin and porous skin layer membrane due its high permeability coefficient which able to generate high lysozyme transmission. It can be categorized as typical ultrafiltration membrane while UF 17 and UF 19 membranes were categorized as tight ultrafiltration membranes. Increased the polymer concentration raised the thickness of membrane support layer and the presence of microvoids was promoted a denser membrane which led to the reduction of the permeability coefficient. Therefore UF 17 and UF 19 needed a high pressure

Table 2 Types of PES UF membrane and its permeability

\begin{tabular}{ccc}
\hline $\begin{array}{c}\text { Membrane } \\
\text { ID }\end{array}$ & $\begin{array}{c}\text { Polymer } \\
\text { Concentration } \\
(\%)\end{array}$ & $\begin{array}{c}\text { Permeability } \\
\text { coefficient }\left[\times \mathbf{1 0} \mathbf{0}^{-6}\right] \\
\left(\mathbf{m}^{\mathbf{3}} / \mathbf{m}^{\mathbf{2}} \mathbf{. s . b a r}\right)\end{array}$ \\
\hline UF 15 & 15 & 10.9 \\
UF 17 & 17 & 1.82 \\
UF19 & 19 & 0.44 \\
\hline
\end{tabular}

to wet the membrane in order to improve the rate of permeability.

\subsection{Membrane Morphology}

In this study, all the fabricated membranes display asymmetric structures with a combination of two layers: active layer and supporting layer and both layers have significant role in thembrane transport property. The cross-section morphology of UF membranes observed by SEM is depicted in Figure 2. Exception to the UF 19, all the prepared membranes are porous due to its regular finger voids structure [17]. UF 15 membrane shows a large microporous finger like structure which indicated that this membrane posses a high porosity. At lower polymer concentration, nonsolvent concentration in the dope solution was higher. Therefore, the non-solvent diffusion into the membrane was higher and the phase separation velocity leads to the formation of big finger like pores in the membranes as can be seen in UF 15 membrane. Large finger like structure which performed from top layer to the bottom layer and a very thin supporting layer of UF 15 was increased its porosity and permeability. A little or extra water content also influence the active layer structure. Thus, both the water content and polymer weight percentage influence the membrane morphology. Besides, the solvent exchange occurred inmediately when the membrane was immersed in water, thus promoted the formation of larger finger-like pores from the processes of polymerlean phase growth and coalescence. On top of that, lower polymer concentration membranes exhibited few sponge structures which resulted from the rapid solvent precipitation during phase inversion process [18].

UF 17 membrane displayed tiny and micropore finger like structure with fine tuned arrangement. The increase in polymer concentration at a constant solvent ratio produced higher solution viscosity and selectivity but generally provided a lower flux. In addition, these condition affected by the thicker selective skins and transition layers caused by the slower redissolution of initial phase outermost separated regions of nascent membranes from an underlying homogeneous solution during dry phase separation [19]. This type of membrane 


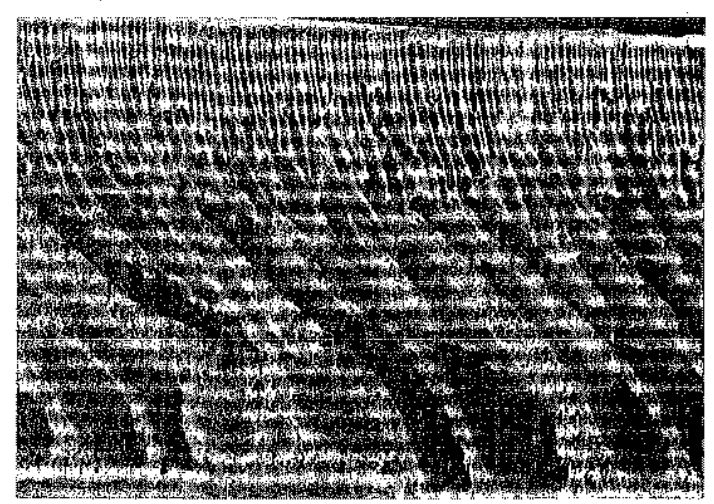

(a)

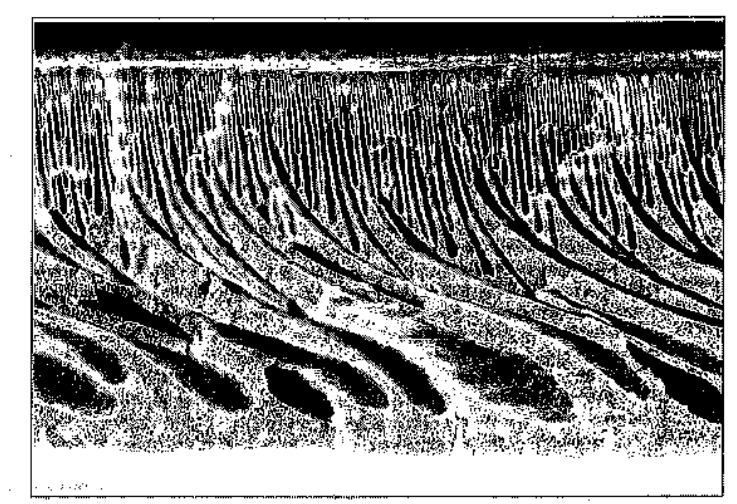

(b)

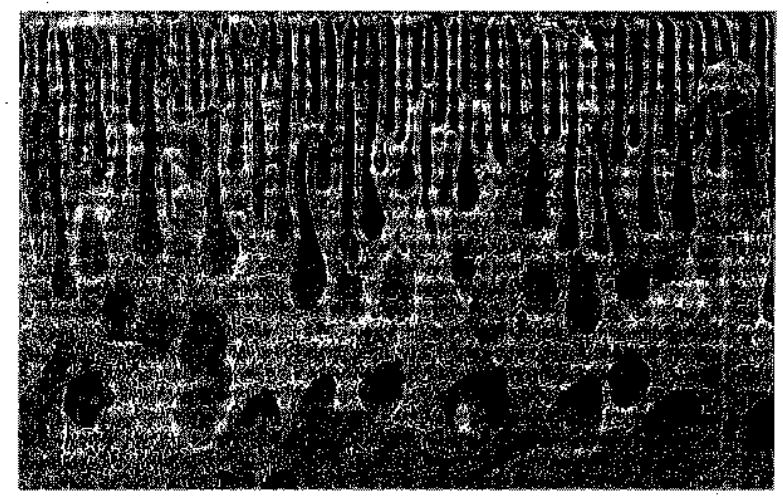

(c)

Figure 2. SEM micrograph for (a) UF 15, (b) UF 17 and (c) UF 19

performed a lower water flux permeability which indicated that the pores presented in this membrane are smaller and did not suitable for protein separation and purification.

UF 19 membrane presents the densest skin layer compared to the other fabricated membranes. UF 19 consisted of high polymer concentration which enhanced the viscosity of the dope solution leading to the formation of smaller pore size. This phenomenon occurs since high viscosity would avoid the diffusion exchange rate of solvent and non-solvent in sub-layer inducing fast phase separation at skin layer and slows the precipitation rate of the sub-layer. Hence, this results in the formation of asymmetric membrane with dense and thick skin layer supported by a closed cell sublayer [20]. Besides, higher polymer concentration was induced the chain entanglement and therefore reduced the formation of the macrovoid in the skin layer [21]. The separation ability of the membrane will be increased but the permeability rate will be declined. Thus, this membrane was claimed to be unsuitable for lysozyme separation since it just retained the lysozme molecule onto the membrane surface instead of allowed it to pass through.

From the observation, it is proved that different polymer weights content and non solvent water quantity made in the mixture ternary dope was promoted different membrane morphology and performance. UF 15 shows the most suitable membrane structural and morphology for application of lysozyme separation and purification.

\subsection{Determination of Molecular Weight Cut Off (MWCO)}

Molecular weight cut off (MWCO) is customarily used to indicate the pore size of ultrafiltration 


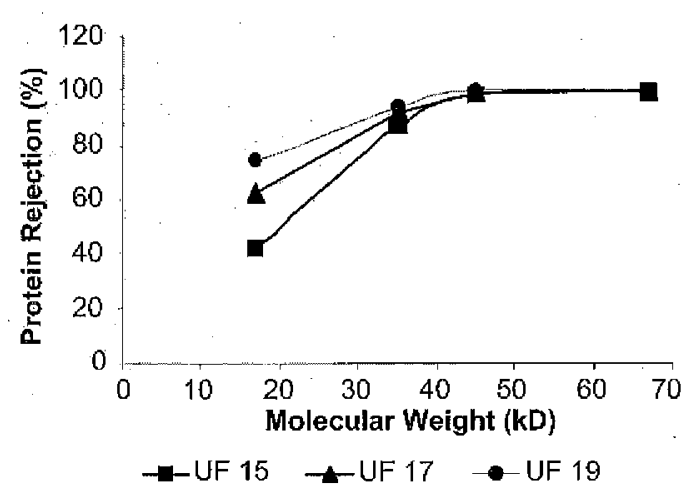

Figure 3 Rejection of different molecular weight proteins in three UF membranes

membrane, and its value can be determined from the solute rejection of membranes against the stable molecules with various weight, which can be measured with ultrafiltration process [22]. The retention rate shown in Figure 3 ascends with the increase of solute molecular weight. Here, the value of $90 \%$ is a standard level to determine whether or not the solid are successfully removed [23]. The molecular weight cut off of UF 15 membrane was estimated to be $43 \mathrm{kD}$ at the point where the rejection is $90 \%$ which is a standard as mentioned above. Increased the polymer concentration to $17 \%$ (UF 17) and $19 \%$ were reduced the molecular weight cut off of these typical membrane to $38 \mathrm{kD}$ and $33 \mathrm{kD}$, respectively. In other words, increases the polymer concentration would reduce the membrane molecular weight cut off. Lysozyme would be easily pass through the UF 15 memibrane due to its larger MWCO which tend to improve the filtrate flux and reduced the fouling. This result might be explained in term of water content in the membrane solution. Increased the polymer concentration would decrease the water content in the dope solution, consequently reduced the rapidness of precipitation in the membrane during wet phase separation in coagulation bath. Thus, a denser membrane with smaller MWCO would produce.

\subsection{Membrane Zeta Potential}

In this study, the UF15-M was chosen as an optimize membrarie for lysozyme separation. This type of membrane was further characterized to determine its zeta potential. UF15-M was observed as negatively charge membrane since its zeta potential was about $-62 \mathrm{mV}$. The other fabricated membranes were estimated to be negatively charged membrane since they were prepared from the same material. Negatively charge membrane are widely used since it can selectively partition ions in the salt mixture through the electrostatic interaction between ions and membrane [24]. In this study, this negatively charge membrane would attract the positively charge lysozyme onto the membrane surface and membrane pores. Operating pressure applied would desorb the lysozyme molecule to pass through the membrane pores which tend to improve the filtrate flux and lysozyme transmission. The other types of fabricated membranes were assumed to be negatively charge also since the came from the same materials.

\subsection{Lysozyme Separation Perfomance}

Figure 3 and 4 represent the flux and lysozyme transmission through the fabricated membrane with different polymer concentration. For each membrane, the flux increased with applied pressure from 2 to 10 bars. The results induced that the lysozyme separation performance was decreased in order of UF $15>$ UF $17>$ UF 19 respectively. Higher permeability was observed at lower polymer concentration membrane due to the presented of larger pore size. Optimum flux and lysozyme transmission were predicted to be at

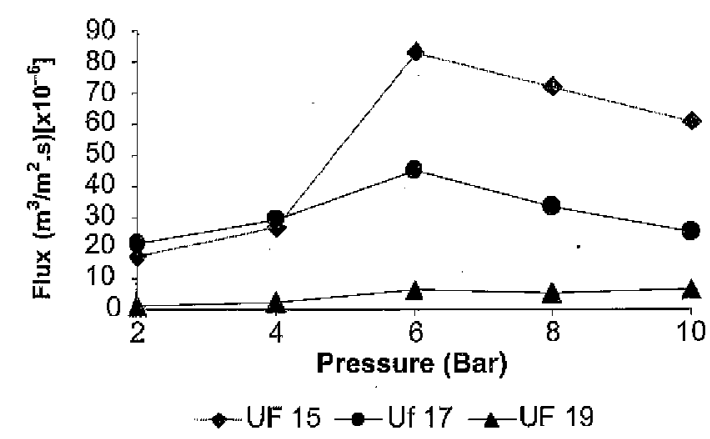

Figure 4 Filtrate flux of lysozyme transmission through three types of UF membranes 
pressure of 6 bars since the highest point for flux and transmission obtained within this operating pressure. After 6 bars, flux and transmission generally decreased and this reduction might due to the fouling and pore blocking phenomena.

At an optimum pressure of 6 bars, UF 15 performed the greatest lysozyme transmission, around $94 \%$. This high transmission was parallel with high flux for about $8.32 \times 10^{-5} \mathrm{~m}^{3} / \mathrm{m}^{2}$.s. Further increase of applied pressure caused the transmission reduced to $86 \%$. and $66 \%$ at pressure 8 and 10 bars, respectively. This phenomenon has occurred since the most lysozyme molecule can pass through the membrane during the initial stage of filtration process when the pore size of membrane is larger than the pore radius of lysozyme. After it reached the saturating point, lysozyme starts to deposit onto the membrane surface and pore wall which leading to enhance fouling and lowered the permeate flux and lysozyme transtnission even further increase of pressure has been done.

UF 17 shows very low tiltrate flux and lysozyme transmission at optimum pressure for about $6.1 \times 10^{-6} \mathrm{~m}^{3} / \mathrm{m}^{2}$.s and $45 \%$, respectively. A small range of lysozyme transmission, between $21-45 \%$ was found within the permeation test of this UF 17. A fine and high density of finger like structure of UF 17 just allowed some of lysozyme molecule to transmit. Most of lysozyme would retain onto the membrane surface and its accumulation was promoted a fouling and cake formation which consequently reduced the flux and transmission. Therefore this membrane was determined to be

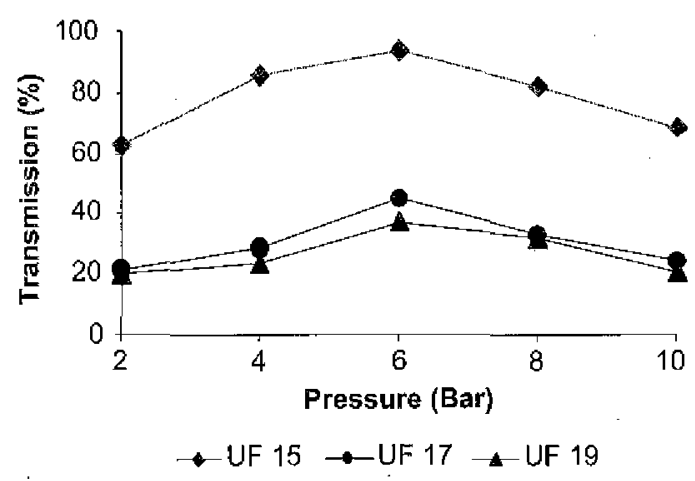

Figure 5 Transmission of protein through three types of UF membranes unsuitable for lysozyme purification due to its low flux and lyzozyme transmission.

UF 19 provided the lowest flux and transmissionat optimum pressure, around $3.9 \times$ $10^{-6} \mathrm{~m}^{3} / \mathrm{m}^{2} \mathrm{~s}$ and $37 \%$, respectively, compared to UF 15 and UF 17. Lysozyme transmission was ranged only between $19-37 \%$. A well arranged finger like structure and spongy support layer supposed to retain the lysozyme molecule onto the membrane surface. The reason for this unreliable result was not clear yet. However, this membrane also could not be claimed as the best membrane for lysozyme purification since it posses a tight structure and low llux which can reduce the lysozyme separation ability in the real application and promote a critical fouling.

The membrane selectivity is basically depended on transport through the pore in skin layer with appropriate pressure where the membrane morphology and membrane properties greatly affected the mechanisms. Concentration polarization could be ignored due to surface stirrer near the membrane surface. However for UF 15 , flux declined occurred between 8 to 10 bars was reduced the lysozyme transmission. It can be assumed that irreversible fouling occurs and clogging in the pores at the higher pressure ware occurred in this case. Since UF 15 posses a high lysozyme transmission along with suitable permeate flux, this type of membrane was assumed to be the optimum membrane for lysozyme separation in this study.

\subsection{CONCLUSION}

Asymmetric UF membranes with different polymer concentration were successfully developed via a simple dry/wet phase inversion technique. The findings of this study proves that the polymer concentration was greatly influenced the membrane performance and morphology. Increased the polymer concentration produced a thicker and denser membrane surface which led to the reduction of flux and lysozyme transmission. Based on the experimental data, UF 15 membrane is seems to be an optimum polymer concentration in preparing an outstanding performance of lysozyme separation process. 


\section{ACKNOWLEDGEMENTS}

The authors wish to express their sincere gratitude to the Ministry Of Science, Technology And Innovation (MOSTI), for the grant of the EScience Fund and Miss S. Maari for their cooperation and support.

\section{REFERENCES}

[1] Ghosh, R. 2002. Review: Protein Separation using Membrane Chromatography: Opportunities and Challenges. Journal of Chromatography A. 952: 13-27.

[2] Cheryan, 1986. Ultrafiltration Handbook. Technomic Publication Co. Lancaster, USA.

[3] Reis, R. V. and A. Zydney. 2007. Bioprocess Membrane Technology. Journal of Membrane Science. 297: 16-50.

[4] Zydney A. L., R. Kuriyel. 2000. Methods in Biotechnology: Downstream Protein Processing. Totowa: Humana Press.

[5] Mulder, M. 1996. Basic Principles of Membrane Technology. $2^{\text {nd }}$ Edition. Kluwer Academic Publishers, The Netherlands.

[6] Idris, A., M. Y. Noordin, A. F. Ismail and S. J. Shilton. 2002. Study of Shear Rate Influence on the Performance of Cellulose Acetate Reverse Osmosis Hollow Fiber Membranes. Journal of Membrane Science. 205: 223237.

[7] Field, R. W., K. F. Md Yunos and Z. F. Cui. 2009. Separation of Proteins using Sandwich Membranes, Desalination. 245: 597-605.

[8] Mark, R. W. and S. Chellam. 1999. Environmentral Science and Technology. American Chemical Society. 17(33): 360366.

[9] Bumsulk, J. 2004. Preparation of Hydrophilic Polyacrylonitrile Blend Membranes for Ultrafiltration. Journal of Membrane Science. 229: 129-136.

[10] Shi, Q., Y. Su, S. Zhu, C. Li, Y. Zhaoa, and Z. Jiang. 2007. A Facile Method for Synthesis of Pegylated Polyethersulfone and its Application in Fabrication of Antifouling Ultrafiltration Membrane. Journal of Membrane Science. 303: 204-212.
[11] Wang, D. L., K. Li, S. Sourirajan, and W. K. Teo, 1993. Phase Separation Phenomena of Polysulfone/solvent/organic non-solvent and Polyethersulfone/solvent/ organic non-solvent Systems. Journal of Application Polymer Science. 50: 1693.

[12] Kesting, R. E. 1985. Synthetic Polymeric Membranes a Structural Perspective. New York: John Wiley and Sons. 31-33.

[13] Ghosh R. and Z. F. Cui. 2000. Protein Purification by Ultrafiltration with Pretreated Membrane. Journal of Membrane Science. 167: 47-53.

[14] Ehsani, N., S. Parkkinen and M. Nyström. 1997. Fractionation of Natural and Model Egg-white Protein Solutions with Modified and Unmodified Polysulfone Ultrafiltration Membranes. Journal of Membrane Science. 123: 105-119.

[15] Evrim, B. A., T. Nalan, N. Candan and D. Adil. 2007. Use of Magnetic Poly(glycidyl methacrylate) Monosize Beads for the Purification of Lysozyme in Batch System. Journal of Chromatography B. 853: 105-113.

[16] Wan, Y. H., J. R. Lub and Z. F. Cui. 2006. Separation of Lysozyme from Chicken Egg White using Ultrafiltration. Separation and Purification Technology. 48: 133142 .

[17] Brown, P. J., S. Ying and J. Yang. 2002. Morphological Structure of Polyetherketone Membranes for Gas Separation Prepared by Phase Inversion. AUTEX Research Journal 2: 101-108.

[18] Young, T. H., L. W. Chen. 1995. Pore Formation Mechanisms of Membranes from Phase Inversion Process. Journal of Desalination. 103: 233-247.

[19] Ahmad, A. L., M. Sarif and S. Ismail. 2005. Development of an Integrally Skinned Ultrafiltration Membrane for Wastewater Treatment: Effect of Different Formulations of PSf/NMP/PVP on Flux and Rejection. Desalination.179: 257-263.

[20] Kim, J. H. and K. H. Lee. 1998. Effect of PEG Additive on Membrane Formation by Phase Inversion, Journal of Membrane Science. 138: 153-163. 
[21] Kimmerle, K. and Strathmann, H. 1990. Analysis of the Structure Determining Process of Phase Inversion Membranes, Desalination. $79: 283-302$.

[22] Becht, N. O., D. J. Malik and E. S. Tarleton. 2008. Evaluation and Comparison of Protein Ultrafiltration Test Results: Dead End Cell Compared with a Cross Flow System. Separation and Purification Technology. 62: 228-239.
[23] Kulkarni, S. S., E. W. Funck, N. N. Li, in Winston W. S. Ho, K. K. Sirkar, (Eds). 1992 Membrane Handbook. New York: van Nostrand Reinhold. 393-394.

[24] Wang, K. Y. and T. S. Chung. 2005. The Characterization of Flat Composite Nanofiltration Membranes and their Applications in the Separation of Cephalexin. Journal of Membrane Science. 247: 37-50. 